\title{
Does Spin-Orbit Coupling Effect Favor Planar Structures for Small Platinum Clusters?
}

\author{
Ali Sebetci快 \\ IFW Dresden, Helmholtzstrasse 20, 01069 Dresden, Germany
}

(Dated: November 3, 2018)

\begin{abstract}
We have performed full-relativistic density functional theory calculations to study the geometry and binding energy of different isomers of free platinum clusters $\mathrm{Pt}_{n}(n=4-6)$ within the spin multiplicities from singlet to nonet. The spin-orbit coupling effect has been discussed for the minimum-energy structures, relative stabilities, vibrational frequencies, magnetic moments, and the highest occupied and lowest unoccupied molecular-orbital gaps. It is found in contrast to some of the previous calculations that 3-dimentional configurations are still lowest energy structures of these clusters, although spin-orbit effect makes some planar or quasi-planar geometries more stable than some other 3-dimentional isomers.
\end{abstract}

PACS numbers: 36.40.Cg, 71.15.Nc, 82.33.Hk

Keywords:

*Electronic address: a.sebetci@ifw-dresden.de 


\section{INTRODUCTION}

Small transition metal clusters have been attracting wide interest due to their potential applications as building blocks of functional nanostructured materials, electronic devices, and nanocatalysts [1]. In particular, platinum is one of the most important ingredients in the heterogeneous catalysis of hydrogenation as well as in the catalysis of the $\mathrm{CO}, \mathrm{NO}_{x}$, and hydrocarbons. It is currently the preferred oxidation and reduction catalyst for lowtemperature PEM fuel cells [2]. As the size of the Pt particles decrease, their catalytical activities tend to increase because of the increased surface areas of smaller particles and the structural sensitivity of some reactions [3].

Lineberger and co-workers have investigated the electronic spectra of small platinum and palladium $(n=2,3)$ clusters by the negative ion photodetachment spectroscopic method [4, 5] and Eberhardt and co-workers have obtained the valence and core-level photoemission spectra of mass selected monodispersed $\mathrm{Pt}_{n}(n=1-6)$ clusters [6]. In the recent theoretical calculations, we have studied bare and hydrogenated $\mathrm{Pt}_{n} \mathrm{H}_{m}(n=1-5 m=0-2)$ clusters in a scalar-relativistic density functional theory (DFT) formalism [7]. A relevant literature can be found in the reference [7] for the previous experimental and theoretical investigations. In addition to them, Saenz et al [8] have worked on the interaction of Pt clusters with molecular oxygen. Futschek et al [9] have presented ab initio density functional studies of structural and magnetic isomers of $\mathrm{Ni}_{n}$ and $\mathrm{Pt}_{n}$ clusters with up to 13 atoms. Seivane and Ferrer [10] have analyzed the impact of the magnetic anisotropy on the geometric structure and magnetic ordering of small atomic clusters of palladium, iridium, platinum, and gold from two to five, six, or seven atoms, depending on the element. Bhattacharyya and Majumder [11] have reported the growth pattern and bonding trends in $\mathrm{Pt}_{n}(n=2-13)$ clusters and concluded in their first principles study that small $\mathrm{Pt}_{n}$ clusters have planar geometries and a structural transition to non-planar geometries occur at $n=10$. Similarly, Huda et al [12] predicted that spin-orbit (SO) coupling effect favors planar structures for small Pt clusters.

In the present work, we discuss the effect of SO coupling on the structural, electronic and magnetic properties of small $\mathrm{Pt}_{n}(n=4-6)$ clusters by employing Gaussian atomic-orbital methods in the full-relativistic DFT. The possible local minima and ground state isomers, binding energies (BE), relative stabilities, magnetic moments, and the highest occupied and the lowest unoccupied molecular-orbital (HOMO-LUMO) energy gaps have been calculated 
with and without SO coupling to compare the results for both cases. Vibrational frequency calculations for each optimized structure has been carried out to differentiate local minima from transition states.

\section{COMPUTATIONAL METHOD}

NWChem 5.0 program package [13] has been used to perform geometry optimizations and total energy calculations by DFT. CRENBL [14] basis set, effective core potential and $\mathrm{SO}$ operator for Pt have been employed where the outer most 18 electrons of free $\mathrm{Pt}$ atom

$\left(5 \mathrm{~s}^{2} 5 \mathrm{p}^{6} 5 \mathrm{~d}^{9} 6 \mathrm{~s}^{1}\right)$ are treated as valence electrons. The generalized gradient approximation of Becke's exchange functional [15] and Lee-Yang-Parr correlation functional [16] (B3LYP) has been chosen as the hybrid exchange-correlation (xc) functional. Wave function and geometry optimization convergence criteria were not worse than the default NWChem criteria. The geometries have been relaxed without imposing any symmetry constraints. Spin-polarized calculations have been done for the first five spin multiplicities (from singlet to nonet). Figures were produced by ChemCraft graphics program [17.

\section{RESULTS AND DISCUSSION}

\section{A. Pt and $\mathrm{Pt}_{2}$}

First, we discuss the properties of $\mathrm{Pt}$ and $\mathrm{Pt}_{2}$ to assess the accuracy of our theoretical method. The ground-state Pt atom was found in the triplet state $\left(5 \mathrm{~d}^{9} 6 \mathrm{~s}^{1}\right)$ for the non-SO coupling case in agreement with the experimantal results [18. The excitation energy of the singlet $5 \mathrm{~d}^{10} 6 \mathrm{~s}^{0}$ state has been calculated as $0.510 \mathrm{eV}$ (neglecting SO coupling) which can be compared with a spin-averaged experimental value of $0.478 \mathrm{eV}[18$. By including $\mathrm{SO}$ effects, the excitation energy of the closed-shell configuration is $0.881 \mathrm{eV}$ to be compared with the experimental value of $0.761 \mathrm{eV}$ [18]. Both of these calculated excitation energies are much better than the ones obtained by Fortunelli in 1999 [19]. We have calculated the SO splitting in the $5 \mathrm{~d}$ orbital of $\mathrm{Pt}$ atom in its singlet state $\left(5 \mathrm{~d}^{10} 6 \mathrm{~s}^{0}\right)$ for simplicity and obtained as $1.131 \mathrm{eV}$, which is in a reasonable agreement with the value of $1.256 \mathrm{eV}$ calculated by the all-electron full-relativistic DFT code FPLO [20] by employing Perdew-Wang [21] local $\mathrm{xc}$ functional in both programs. The ionization potential (IP) of Pt has been calculated as 
$9.319 \mathrm{eV}$ in the full-relativistic case where the experimental value is $8.958 \mathrm{eV}$ [22]. Huda et al has calculated IP of Pt as $9.381 \mathrm{eV}$ in their DFT study with the projected augmented wave (PAW )method [12]. The calculated electron affinity is $1.806 \mathrm{eV}$ which is comparable to the experimental value of $2.123 \pm 0.001 \mathrm{eV}[23$. The discrepancy between the calculated and experimental values of the electron affinity may be reduced when the experimental value is improved by $\mathrm{SO}$ effects which can be estimated to decrease it by about $0.2 \mathrm{eV}[24]$.

The bond lengths for the Pt dimer were found to be $2.373 \stackrel{\circ}{A}$ and $2.406 \stackrel{\AA}{A}$ for non-SO and SO calculations respectively, while the experimental value [25] is $2.333 \AA$. The corresponding binding energies are $2.708 \mathrm{eV}$ (non-SO) and $2.476 \mathrm{eV}$ (SO), while the experimental value is $3.14 \pm 0.02 \mathrm{eV}$ [26]. Thus, the present method slightly overestimates the Pt dimer bond length and therefore underestimates the binding energy. Both of the calculated vibrational frequencies of $237 \mathrm{~cm}^{-1}$ (non-SO) and $219 \mathrm{~cm}^{-1}$ (SO) agree pretty well with the measured value of $223 \mathrm{~cm}^{-1}$ [27].

\section{B. $\mathrm{Pt}_{4}$}

We report the relative stability of $\mathrm{Pt}_{4}$ isomers with and without $\mathrm{SO}$ coupling for different spin multiplicities in Table I. The geometric structures and bond lengths of each isomer of $\mathrm{Pt}_{4}$ for their most stable spin multiplicities are given in Fig. 1 .

Similar to the results in our previous study on bare and hydrogenated small Pt clusters [7] where we did not employ SO coupling effects and to the references [9, 28], the calculated lowest energy structure of Pt tetramer is a tetrahedron for both cases of non-SO and SO. When SO effects are not considered, the ground state spin multiplicity of the tetrahedron is 3 and it has the point group symmetry $C_{3 v}$. On the other hand, when SO effects are taken into account, quintet, septet, and nonet initial spin multiplicities converge to the same spin moment of $3.64 \mu_{B}$ and this state has $26 \mathrm{meV}$ lower energy than the one with $1.84 \mu_{B}$ spin moment. In addition, the point group symmetry of the optimized structure in this case is $D_{2 d}$. Our prediction of tetrahedron as the ground state geometry is in contrast with some of the previous results [11, 12, 29]. A common feature of these studies is that they have been done by employing plane wave codes (references [11, 12] are by PAW method of VASP [30], reference [29] is by CPMD [31]) and predict that the rhombus isomer is the lowest energy structure with a spin multiplicity of 5 despite the fact that Futschek et al [9] have employed 
the code VASP too and obtained a distorted tetrahedron similar to our results. According to the present calculations of non-SO case, the quintet state is the ground magnetic state of the rhombus, but its total energy is $0.206 \mathrm{eV}$ higher than that of the tetrahedron. For the SO case, the rhombus whose singlet, triplet, quintet, and septet initial spin multiplicities converge to a spin moment between 2.08 and $2.11 \mu_{B}$ has $0.133 \mathrm{eV}$ higher energy than the tetrahedron. The optimized structures of the rhombus in both non-SO and SO cases have the same point group symmetry $C_{2 v}$. As in the case of the dimer, SO effects slightly stretch the bond lengths. Both of these structures are out off plane where the angles between the triangular planes are $113^{\circ}$ (non-SO) and $105^{\circ}$ (SO). Thus, SO effect strengthens the nonplanarity of the rhombus in contrast to the findings of Huda et al [12]. The third, and fourth lowest energy isomers of Pt tetramer are the square and Y-like (see Fig. 1) planar structures, respectively. The total energy of the singlet square (non-SO) is $0.232 \mathrm{eV}$ higher than that of the global minimum. SO effect increases this energy difference to a value of $0.357 \mathrm{eV}$ which is dissimilar to the general trend that SO effects decrease the energy differences between the isomers. For the Y-like isomer, the energy seperations from the lowest energy structure are $0.551 \mathrm{eV}$ (non-SO quintet) and $0.337 \mathrm{eV}\left(\mathrm{SO} 3.47 \mu_{B}\right)$.

\section{C. $\mathbf{P t}_{5}$}

We have identified six different stable isomers of $\mathrm{Pt}_{5}$ clusters and reported their relative stabilities in Table II and their structures and bond lengths in Fig. 2. Although a bridge side capped tetrahedron with a spin multiplicity of 5 has been obtained as the lowest energy structure for the non-SO case which agrees with our previous calculations [7], SO coupling effects favor a pyramid with $5.58 \mu_{B}$ spin moment. The energy seperation between the capped tetrahedron and the pyramid in the former case is only $11 \mathrm{meV}$ which may be considered within the accuracy of the calculations. On the other hand, SO effects favor pyramidal structure against the tetrahedral one as much as $167 \mathrm{meV}$. While the obtained pyramidal isomer as the lowest energy structure contradics the findings of Bhattacharyya and Majumder [11], Huda et al [12], Grönbeck and Andreoni [29], Xiao and Wang [32], Saenz et al [8], and Futschek et al [9], it agrees with the predictions of Balasubramanian et al [28], Yang et al [33], and Seivane and Ferrer [10]. Bhattacharyya and Majumder [11], and Huda et al [12] have predicted planar bridge side capped structure, Grönbeck and Andreoni [29], and 
Saenz et al [8] have predicted planar W-like structure, Xiao and Wang [32], and Futschek et al [9] have predicted 3-dimensional trigonal bipyramid as the global minimum configuration.

The third low lying isomer is an out of plane W-like structure (see Fig. 22) with a spin moment of $3.58 \mu_{B}$ having $173 \mathrm{meV}$ higher energy than the global minimum. Previously we have identified this trapezoidal-type structure as the fifth low lying isomer [7]. However, in that study we have considered only its singlet and triplet states. Thus, the discrepancy can be attributed to the limitation of the previous calculations for the first two spin multiplicities. The fourth energetically favorable structure is a bipyramid with $3.57 \mu_{B}$ spin moment. The planar, bridge side capped square structure which has been predicted as the global minimum of $\mathrm{Pt}_{5}$ clusters [11, 12] by a plane wave code, has been obtained as the fifth isomer in our calculations. It has $0.674 \mathrm{eV}$ (non-SO) and $0.373 \mathrm{eV}(\mathrm{SO})$ higher energies than the lowest energy structures. Finally, a non-planar X-like gometry has been identified as a stable isomer with the highest total energy. Unlike most of the other isomers, the most stable magnetic state of the X-like structure for SO case could be obtained from an initial nonet state which gives very high relative energy $(2.859 \mathrm{eV})$ in non-SO calculations.

The effect of SO coupling on the bond lengths of $\mathrm{Pt}_{5}$ clusters is not monotonic. For the pyramidal structure, while the interatomic distances between the apex atom and the atoms on the squared plane are stretched, the distances between the atoms on the squared plane are diminished. For the capped tetrahedron, as SO effects make the triangle constructed by the capping atom and the two atoms bonded to the capping atom a bit larger, all other bond lengths are kept nearly the same. All bond lengths of W-like structure and most of them in X-like structure are stretched by SO coupling, on the contrary, it does not have a significant effect on the bond length of the bipyramid, and even causes contractions in the bond length of the capped square isomer.

\section{D. $\mathbf{P t}_{6}$}

The relative stabilities of eleven different isomer of $\mathrm{Pt}_{6}$ can be found in Table III. Their bond lengths and structures have been given in Fig. 3. The most stable structure of $\mathrm{Pt}_{6}$ has been obtained as a trigonal prism in the septet state for non-SO case and a state with 5.43 $\mu_{B}$ spin moment for SO case. Xiao and Wang [32] have predicted a planar double capped square, Bhattacharyya and Majumder [11] have identifed a planar triangular structure, 
Futschek et al [9] have obtained a face capped pyramid as the lowest energy isomer of $\mathrm{Pt}_{6}$. In our calculations, the triangle is the second lowest energy structure having $0.113 \mathrm{eV}$ high energy (SO case) than the prism, the face capped pyramid is the third isomer with 0.154 eV higher energy, while an out-off plane double capped square has been obtained as the last isomer with $0.696 \mathrm{eV}$ relative energy to the lowest one. Dissimilar to $\mathrm{Pt}_{4}$ and $\mathrm{Pt}_{5}$ clusters, $\mathrm{SO}$ coupling effect changes the order of the most $\mathrm{Pt}_{6}$ isomers. When $\mathrm{SO}$ effect does not included in the calculations, the second and the third isomers are predicted as the face capped pyramid and the bridge capped bipyramid, respectively. Similarly, 3-dimensional double capped tetrahedron has less relative energy for non-SO case than SO case. On the other hand, SO effect decreases significantly the relative energies of planar or quasi-planar structures. For instance, $0.390 \mathrm{eV}$ of septet triangle becomes $0.113 \mathrm{eV}, 2.031 \mathrm{eV}$ nonet double square becomes $0.334 \mathrm{eV}, 0.880 \mathrm{eV}$ of double capped square becomes $0.696 \mathrm{eV}$ due to $\mathrm{SO}$ mixing. The big difference between the relative energies of double square in the nonet state (2.031 eV for non-SO case and $0.334 \mathrm{eV}$ for $\mathrm{SO}$ case) can be explained by the fact that $\mathrm{SO}$ effect highly changes the initial spin multiplicity and converges to a spin moment of $0.74 \mu_{B}$. Thus, we agree with Huda et al [12] that SO coupling do has a considerable effect on these clusters, and it increases the stability of planar structures. However, we do not agree with neither Huda et al [12] nor Bhattacharyya and Majumder [11] that the planar structures are the most stable isomers of small $\mathrm{Pt}_{n}(n=4-6)$ clusters. This conclusion is supported by not only the results of Futschek et al [9] but also the findings of Tian et al [34], who have calculated the structural properties of $\mathrm{Pt}_{7}$ cluster by using DFT with both Gaussian and plane wave basis sets and obtained a 3-dimentional coupled tetragonal pyramid as the global minimum, which can be constructed by adding an atom on the center of one of the rectangular faces of the triangular prism. As in the case of $\mathrm{Pt}_{5}$, while $\mathrm{SO}$ effect increases some of the bond lengths of $\mathrm{Pt}_{6}$ clusters, it decreases some others.

\section{E. Most Stable Isomers of $\mathbf{P t}_{4}-\mathbf{P t}_{6}$}

Point group symmetries, spin magnetic moments, total binding energies per atom, SO energies per atom (binding energy difference between non-SO and SO cases), HOMO-LUMO gaps, and lowest and highest vibrational frequencies for the most stable spin states of each isomers of all Pt clusters studied in this work can be found in Table IV. SO coupling effect 
always reduces binding energy since it makes a larger contribution to the atomic energy than to the cluster energy. As the cluster size increases from 4 to 6 , the binding energy per atom increases as well (from $2.122 \mathrm{eV}$ to $2.483 \mathrm{eV}$ for non-SO case, from $1.914 \mathrm{eV}$ to 2.286 $\mathrm{eV}$ for $\mathrm{SO}$ case). In contrast to the results of Huda et al [12], SO energy per atom decreases from $0.208 \mathrm{eV}$ to $0.193 \mathrm{eV}$ when the size of the clusters changes from 4 to 5 . For the lowest energy structure of $\mathrm{Pt}_{6}$, SO energy per atom is $0.196 \mathrm{eV}$. Except the fisrt isomers of $\mathrm{Pt}_{5}$ and $\mathrm{Pt}_{6}$ and the seventh isomer of $\mathrm{Pt}_{6}, \mathrm{HOMO}-\mathrm{LUMO}$ gaps are reduced due to $\mathrm{SO}$ mixing. For the non-SO case, planar or quasi-planar (4-4), (5-5), (5-6), (6-2), (6-4), (6-8) structures have significantly large HOMO-LUMO gaps. SO effects reduce these large gaps. In general, SO coupling effect does not change the vibrational frequencies considerably.

\section{SUMMARY}

In conclusion, we have studied the effect of SO coupling on small Pt clusters, $\mathrm{Pt}_{n}(n=4-6)$. Four isomers of $\mathrm{Pt}_{4}$, six isomers of $\mathrm{Pt}_{5}$, and eleven isomers of $\mathrm{Pt}_{6}$ were calculated with and without SO effects. It is found that SO coupling effects have a considerable effect on these clusters which can change the order of isomers. Although it increases the stability of planar structures, it cannot make these planar structures the most stable isomers. The lowest energy structures of $\mathrm{Pt}_{4}, \mathrm{Pt}_{5}$, and $\mathrm{Pt}_{6}$ clusters are predicted as 3-dimentional tetrahedron, pyramid, and trigonal prism, respectively. In general, SO mixing reduces both total binding energies and HOMO-LUMO gaps.

[1] G. Schmid, Adv. Eng. Mater. 3, 737 (2001).

[2] W. Vielstich, A. Lamm, H.A. Gasteiger, Handbook of Fuel Cells: Fundamentals, Technology, Applications; Wiley: West Sussex, (2003).

[3] Y. Xu, W.A. Shelton, W.F. Schneider, J. Phys. Chem. A, 110, 5839 (2006).

[4] J. Ho, M. L. Polak, K. M. Ervin, W. C. Lineberger, J. Chem. Phys. 99, 8542 (1993).

[5] K. M. Ervin, J. Ho, W. C. Lineberger, J. Chem. Phys. 89, 4514 (1988).

[6] W. Eberhardt, P. Fayet, D. M. Cox, Z. Fu, A. Kaldor, R. Sherwood, D. Sondericker, Phys. Rev. Lett. 64, 780 (1990).

[7] A. Sebetci, Chem. Phys. 331, 9 (2006). 
[8] L.R. Saenz, P.B. Balbuena, J.M. Seminario, J. Phys. Chem. A 110, 11968 (2006).

[9] T. Futschek, J. Hafner, M. Marsman, J. Phys.: Condens. Matter 18, 9703 (2006).

[10] L.F. Seivane, J. Ferrer, Phys. Rev. Lett. 99, 183401 (2007).

[11] K. Bhattacharyya, C. Majumder, Chem. Phys. Lett. 446, 374 (2007).

[12] M.N. Huda, M.K. Niranjan, B.R. Sahu, L. Kleinman, Phys. Rev. A 73, 053201 (2006).

[13] E.J. Bylaska, W.A. de Jong, K. Kowalski, T.P. Straatsma, M. Valiev, D. Wang, E. Apra, T.L. Windus, S. Hirata et al. "NWChem, A Computational Chemistry Package for Parallel Computers, Version 5.0" (2006), Pacific Northwest National Laboratory, Richland, Washington 99352-0999, USA.

[14] R.B. Ross, J.M. Powers, T. Atashroo, W.C. Ermler, L.A. LaJohn, P.A. Christiansen, J. Chem. Phys. 93, 6654 (1990).

[15] A.D. Becke, J. Chem. Phys. 98, 5648 (1993).

[16] C. Lee, W. Yang, R.G. Parr, Phys. Rev. B 37, 785 (1988).

[17] http://www.chemcraftprog.com.

[18] C.E. Moore, Natl Bur. Stand. (US) Circ. 467 (1971).

[19] A. Fortunelli, J. Mol. Struct. (THEOCHEM) 493, 233 (1999).

[20] K. Koepernik, H.Eschrig, Phys. Rev. B 59, 1743 (1999)

[21] J.P. Perdew, Y. Wang, Phys. Rev. B 45, 13244 (1992).

[22] A. Marijnissen, T.T. ter Meulen, P.A. Hackett, B. Simard, Phys. Rev. A 52, 2606 (1995).

[23] N.D. Gibson, B.J. Davies, D.J. Larson, J. Chem. Phys. 98, 5104 (1993).

[24] H. Hotop, W.C. Lineberger, J. Phys. Chem. Ref. Data 14, 731 (1985).

[25] M.B. Airola, M.D. Morse, J. Chem. Phys. 116, 1313 (2002).

[26] S. Taylor, G.W. Lemire, Y.M. Hamrick, Z. Fu, M.D. Morse, J. Chem. Phys. 89, 5517 (1988).

[27] J.C. Fabbi, J.D. Langenberg, Q.D. Costello, M.D. Morse, L. Karlsson, J. Chem. Phys. 115, 7543 (2001).

[28] K. Balasubramanian, J. Chem. Phys. 87, 6573 (1987); D. Dai, K. Balasubramanian, ibid. 103, 648 (1995); D. Majumdar, D. Dai, K. Balasubramanian, ibid. 113, 7919 (2000); D. Majumdar, D. Dai, K. Balasubramanian, ibid. 113, 7928 (2000).

[29] H. Grönbeck, W. Andreoni, Chem. Phys. 262, 1 (2000).

[30] G. Kresse, J. Furthmüller, Comput. Mater. Sci. 6, 15 (1996).

[31] R. Car, M. Parrinello, Phys. Rev. Lett. 55, 2471 (1985). 
[32] L. Xiao, L. Wang, J. Phys. Chem. A 108, 8605 (2004).

[33] S.H. Yang, D.A. Drabold, J.B. Adams, P. Ordejón, K. Glassford, J. Phys.: Condens. Matter 9, L39, (1997).

[34] W.Q. Tian, M. Ge, B.R. Sahu, D. Wang, T. Yamada, S. Mashiko, J. Phys. Chem. A 108, 3806 (2004). 
TABLE I: Relative Stability of $\mathrm{Pt}_{4}$ Isomers Predicted by Scalar-Relativistic (non-SO) and fullrelativistic (SO) DFT calculations.

\begin{tabular}{|c|c|c|c|c|c|}
\hline \multirow[t]{3}{*}{ Isomer } & \multirow[t]{3}{*}{ Structure } & \multicolumn{2}{|c|}{ non-SO } & \multicolumn{2}{|c|}{$\mathrm{SO}$} \\
\hline & & Spin & $\Delta \mathrm{E}$ & Spin & $\Delta \mathrm{E}$ \\
\hline & & Moment $\left(\mu_{B}\right)$ & $(\mathrm{eV})$ & Moment $\left(\mu_{B}\right)$ & $(\mathrm{eV})$ \\
\hline \multirow[t]{4}{*}{$(4-1)$} & Tetrahedron & 2 & 0.000 & 1.84 & 0.026 \\
\hline & & 4 & $0.109^{a}$ & 3.64 & 0.000 \\
\hline & & 6 & 1.110 & 3.64 & 0.000 \\
\hline & & 8 & 2.239 & 3.64 & 0.000 \\
\hline \multirow[t]{4}{*}{$(4-2)$} & Rhombus & 0 & 0.576 & 2.08 & 0.133 \\
\hline & & 2 & 0.471 & 2.11 & 0.133 \\
\hline & & 4 & 0.206 & 2.10 & 0.133 \\
\hline & & 6 & 0.945 & 2.09 & 0.134 \\
\hline \multirow[t]{2}{*}{$(4-3)$} & Square & 0 & 0.232 & 0.00 & 0.357 \\
\hline & & 2 & 0.321 & 1.77 & 0.493 \\
\hline \multirow[t]{4}{*}{$(4-4)$} & Y-like & 0 & 1.410 & 0.54 & 0.410 \\
\hline & & 2 & 0.892 & 1.50 & 0.458 \\
\hline & & 4 & 0.551 & 3.51 & 0.354 \\
\hline & & 6 & 1.134 & 3.47 & 0.337 \\
\hline
\end{tabular}

${ }^{a}$ saddle point 
TABLE II: Relative Stability of $\mathrm{Pt}_{5}$ Isomers Predicted by Scalar-Relativistic (non-SO) and fullrelativistic (SO) DFT calculations.

\begin{tabular}{|c|c|c|c|c|c|}
\hline \multirow[t]{3}{*}{ Isomer } & \multirow[t]{3}{*}{ Structure } & \multicolumn{2}{|c|}{ non-SO } & \multicolumn{2}{|c|}{$\mathrm{SO}$} \\
\hline & & Spin & $\Delta \mathrm{E}$ & Spin & $\Delta \mathrm{E}$ \\
\hline & & Moment $\left(\mu_{B}\right)$ & $(\mathrm{eV})$ & Moment $\left(\mu_{B}\right)$ & $(\mathrm{eV})$ \\
\hline \multirow[t]{4}{*}{$(5-1)$} & Pyramid & 2 & 0.460 & 3.46 & $0.530^{a}$ \\
\hline & & 4 & 0.149 & 3.79 & 0.144 \\
\hline & & 6 & 0.011 & 5.58 & 0.000 \\
\hline & & 8 & 0.816 & 7.28 & $0.757^{a}$ \\
\hline \multirow[t]{4}{*}{$(5-2)$} & Capped Tetrahedron & 0 & 0.920 & 0.00 & 0.855 \\
\hline & & 2 & 0.428 & 2.38 & 0.500 \\
\hline & & 4 & 0.000 & 3.67 & 0.167 \\
\hline & & 6 & 0.336 & 5.18 & 0.278 \\
\hline \multirow[t]{3}{*}{$(5-3)$} & W-like & 0 & 0.661 & 0.00 & 0.609 \\
\hline & & 4 & 0.169 & 3.58 & 0.173 \\
\hline & & 6 & 0.536 & 2.03 & 0.193 \\
\hline \multirow[t]{5}{*}{$(5-4)$} & Bipyramid & 0 & 1.011 & 0.02 & 0.807 \\
\hline & & 2 & 0.319 & 2.05 & $0.339^{a}$ \\
\hline & & 4 & 0.186 & 3.56 & 0.247 \\
\hline & & 6 & 0.609 & 3.57 & $0.247^{a}$ \\
\hline & & 8 & 0.954 & 3.97 & 0.388 \\
\hline$(5-5)$ & Capped Square & 2 & 0.674 & 2.43 & 0.363 \\
\hline \multirow[t]{3}{*}{$(5-6)$} & $\mathrm{X}$-like & 0 & 1.319 & 0.00 & 0.713 \\
\hline & & 2 & 0.930 & 1.83 & 0.524 \\
\hline & & 8 & 2.859 & 3.39 & 0.490 \\
\hline
\end{tabular}

${ }^{a_{\text {saddle point }}}$ 
TABLE III: Relative Stability of $\mathrm{Pt}_{6}$ Isomers Predicted by Scalar-Relativistic (non-SO) and fullrelativistic (SO) DFT calculations.

\begin{tabular}{|c|c|c|c|c|c|}
\hline \multirow[t]{3}{*}{ Isomer } & \multirow[t]{3}{*}{ Structure } & \multicolumn{2}{|c|}{ non-SO } & \multicolumn{2}{|c|}{$\mathrm{SO}$} \\
\hline & & Spin & $\Delta \mathrm{E}$ & Spin & $\Delta \mathrm{E}$ \\
\hline & & Moment $\left(\mu_{B}\right)$ & $(\mathrm{eV})$ & Moment $\left(\mu_{B}\right)$ & $(\mathrm{eV})$ \\
\hline \multirow[t]{3}{*}{$(6-1)$} & Trigonal Prism & 4 & 0.246 & 3.68 & 0.285 \\
\hline & & 6 & 0.000 & 5.43 & 0.000 \\
\hline & & 8 & 0.168 & 5.41 & 0.001 \\
\hline \multirow[t]{4}{*}{$(6-2)$} & Triangle & 2 & 0.923 & 1.63 & 0.219 \\
\hline & & 4 & $0.957^{a}$ & 2.69 & 0.209 \\
\hline & & 6 & 0.390 & 3.44 & 0.113 \\
\hline & & 8 & 1.831 & 3.43 & 0.115 \\
\hline \multirow[t]{3}{*}{$(6-3)$} & Face Capped Pyramid & 2 & 0.404 & 2.50 & 0.333 \\
\hline & & 4 & 0.221 & 4.75 & 0.156 \\
\hline & & 6 & 0.150 & 4.75 & 0.154 \\
\hline \multirow[t]{2}{*}{$(6-4)$} & Double Square & 6 & 0.684 & 5.38 & 0.756 \\
\hline & & 8 & 2.031 & 0.74 & 0.334 \\
\hline \multirow[t]{3}{*}{$(6-5)$} & Bridge Capped Pyramid & 0 & 0.865 & 0.00 & 0.781 \\
\hline & & 6 & 0.644 & 5.42 & 0.338 \\
\hline & & 8 & 0.899 & 5.13 & 0.467 \\
\hline \multirow[t]{3}{*}{$(6-6)$} & Face Capped Bipyramid & 0 & 1.418 & 0.00 & 1.044 \\
\hline & & 2 & 0.598 & $1.74^{b}$ & $0.686^{b}$ \\
\hline & & 8 & 0.369 & $7.01^{b}$ & $0.355^{b}$ \\
\hline \multirow[t]{3}{*}{$(6-7)$} & Octahedron & 0 & 1.326 & 0.00 & 1.620 \\
\hline & & 2 & 1.132 & 4.83 & 0.509 \\
\hline & & 6 & 1.041 & 2.44 & 0.711 \\
\hline \multirow[t]{3}{*}{$(6-8)$} & W-like & 0 & 0.728 & 0.00 & $0.763^{a}$ \\
\hline & & 2 & 1.301 & 4.02 & 0.520 \\
\hline & & 6 & 0.530 & 4.06 & 0.519 \\
\hline \multirow[t]{2}{*}{$(6-9)$} & Double Capped Tetrahedron & 4 & 0.398 & 3.66 & 0.649 \\
\hline & & 6 & 0.782 & 5.08 & 0.678 \\
\hline \multirow[t]{2}{*}{$(6-10)$} & Bridge Capped Bipyramid & 0 & 0.744 & 0.00 & 0.663 \\
\hline & & 4 & 0.349 & $3.54^{b}$ & $0.801^{b}$ \\
\hline \multirow[t]{5}{*}{$(6-11)$} & Double Capped Square & 0 & 0.941 & 1.31 & 0.807 \\
\hline & & 2 & 0.669 & 1.83 & 0.729 \\
\hline & & 4 & 0.728 & 3.22 & $0.696^{a}$ \\
\hline & & 1.6 & 0.880 & 3.22 & 0.696 \\
\hline & & 10 & 1.483 & 3.21 & 0.696 \\
\hline
\end{tabular}


TABLE IV: Isomeric structure properties of $\mathrm{Pt}_{n}(n=4-6)$ clusters with and without SO coupling.

\begin{tabular}{|c|c|c|c|c|c|c|c|c|c|c|c|c|}
\hline & \multirow[t]{2}{*}{ Structure } & \multicolumn{2}{|c|}{ Symmetry } & \multicolumn{2}{|c|}{$\begin{array}{c}\text { Spin } \\
\text { Moment }\left(\mu_{B}\right)\end{array}$} & \multicolumn{2}{|c|}{$\begin{array}{l}\text { Total BE } \\
(\mathrm{eV} / \text { atom })\end{array}$} & \multirow[t]{2}{*}{$\begin{array}{l}\text { SO Energy } \\
(\mathrm{eV} / \text { atom })\end{array}$} & \multicolumn{2}{|c|}{$\begin{array}{c}\text { HOMO-LUMO } \\
\text { gap }(\mathrm{eV})\end{array}$} & \multicolumn{2}{|c|}{$\begin{array}{c}\omega_{l} \text { and } \omega_{h}{ }^{a} \\
\left(\mathrm{~cm}^{-1}\right)\end{array}$} \\
\hline & & non-SO & $\mathrm{SO}$ & non-SO & $\mathrm{SO}$ & non-SO & $\mathrm{SO}$ & & non-SO ${ }^{b}$ & $\mathrm{SO}$ & non-SO & $\mathrm{SO}$ \\
\hline$(4-1)$ & Tetrahedron & $C_{3 v}$ & $D_{2 d}$ & 2 & 3.64 & 2.122 & 1.914 & 0.208 & 1.603 & 1.162 & 80,215 & 99,215 \\
\hline$(4-2)$ & Rhombus & $C_{2 v}$ & $C_{2 v}$ & 4 & 2.10 & 2.071 & 1.882 & 0.190 & 1.890 & 1.433 & 50,213 & 44,206 \\
\hline$(4-3)$ & Square & $D_{4 h}$ & $D_{4 h}$ & 0 & 0.00 & 2.064 & 1.825 & 0.239 & 1.577 & 1.487 & 9,198 & 41,187 \\
\hline$(4-4)$ & Y-like & $C_{s}$ & $C_{1}$ & 4 & 3.49 & 1.985 & 1.830 & 0.155 & 2.316 & 1.903 & 42,234 & 48,241 \\
\hline$(5-1)$ & Pyramid & $C_{2 v}$ & $C_{2 v}$ & 6 & 5.58 & 2.319 & 2.126 & 0.193 & 1.584 & 1.629 & 47,209 & 51,197 \\
\hline$(5-2)$ & Capped Tetra. & $C_{2 v}$ & $C_{s}$ & 4 & 3.67 & 2.321 & 2.090 & 0.231 & 1.656 & 1.403 & 39,212 & 46,198 \\
\hline$(5-3)$ & W-like & $C_{1}$ & $C_{1}$ & 4 & 3.58 & 2.288 & 2.087 & 0.201 & 1.780 & 1.563 & 41,229 & 46,230 \\
\hline$(5-4)$ & Bipyramid & $D_{3}$ & $C_{1}$ & 4 & 3.56 & 2.284 & 2.077 & 0.207 & 1.807 & 1.236 & 71,223 & 69,210 \\
\hline$(5-5)$ & Capped Square & $C_{1}$ & $C_{1}$ & 2 & 2.43 & 2.187 & 2.053 & 0.134 & 1.946 & 1.296 & 50,229 & 50,228 \\
\hline$(5-6)$ & $\mathrm{X}$-like & $C_{1}$ & $C_{1}$ & 2 & 3.39 & 2.135 & 2.028 & 0.107 & 2.063 & 1.754 & 30,269 & 34,246 \\
\hline$(6-1)$ & Trigonal Prism & $C_{2 v}$ & $C_{1}$ & 6 & 5.42 & 2.483 & 2.286 & 0.196 & 1.000 & 1.173 & 19,197 & 11,184 \\
\hline$(6-2)$ & Triangle & $C_{1}$ & $C_{1}$ & 6 & 3.44 & 2.418 & 2.267 & 0.151 & 3.081 & 1.392 & 37,239 & 26,242 \\
\hline$(6-3)$ & Face Capped Pyr. & $C_{1}$ & $C_{2}$ & 6 & 4.75 & 2.458 & 2.260 & 0.198 & 1.339 & 1.319 & 39,206 & 38,201 \\
\hline$(6-4)$ & Double Square & $C_{2 v}$ & $C_{1}$ & 6 & 0.74 & 2.369 & 2.230 & 0.139 & 1.958 & 1.343 & 31,230 & 34,245 \\
\hline$(6-5)$ & Bridge Capped Pyr. & $C_{1}$ & $C_{1}$ & 6 & 5.42 & 2.376 & 2.227 & 0.147 & 1.662 & 1.592 & 32,216 & 22,204 \\
\hline$(6-6)$ & Face Capped Bipyr. & $C_{1}$ & $C_{1}$ & 8 & 7.01 & 2.421 & 2.226 & 0.195 & 2.579 & 1.325 & 35,200 & $c$ \\
\hline$(6-7)$ & Octahedron & $C_{1}$ & $C_{2}$ & 6 & 4.83 & 2.309 & 2.201 & 0.108 & 0.870 & 1.304 & 72,209 & 49,194 \\
\hline$(6-8)$ & W-like & $C_{1}$ & $C_{s}$ & 6 & 4.04 & 2.395 & 2.199 & 0.196 & 2.444 & 1.384 & 36,238 & 47,226 \\
\hline$(6-9)$ & Double Capped Tetra. & $D_{2 d}$ & $D_{2 d}$ & 4 & 3.66 & 2.417 & 2.177 & 0.240 & 1.824 & 1.265 & 28,192 & 27,196 \\
\hline$(6-10)$ & Bridge Capped Bipyr. & $C_{1}$ & $C_{1}$ & 4 & 0.00 & 2.425 & 2.175 & 0.250 & 1.625 & 1.326 & 41,217 & 48,217 \\
\hline$(6-11)$ & Double Capped Square & $C_{2}$ & $C_{1}$ & 2 & 3.22 & 2.371 & 2.170 & 0.202 & 1.511 & 1.279 & 30,216 & 30,229 \\
\hline
\end{tabular}

${ }^{a}$ Lowest and highest vibrational frequencies

${ }^{b} \alpha$ spin

${ }^{c}$ convergence cannot be achieved 

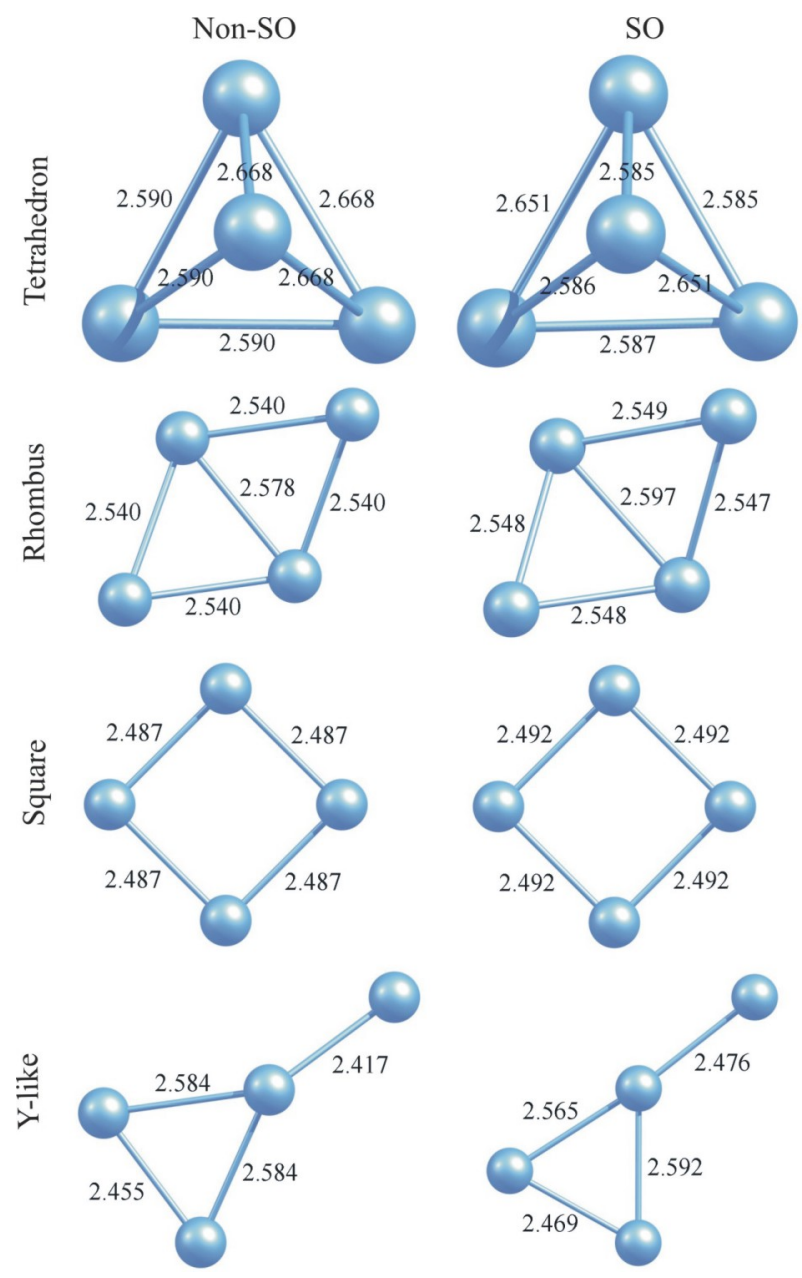

FIG. 1: Relaxed geometries of $\mathrm{Pt}_{4}$ isomers for the most stable spin multiplicity of each isomer with and without SO coupling effects (distances are in $\stackrel{A}{A}$ ). 

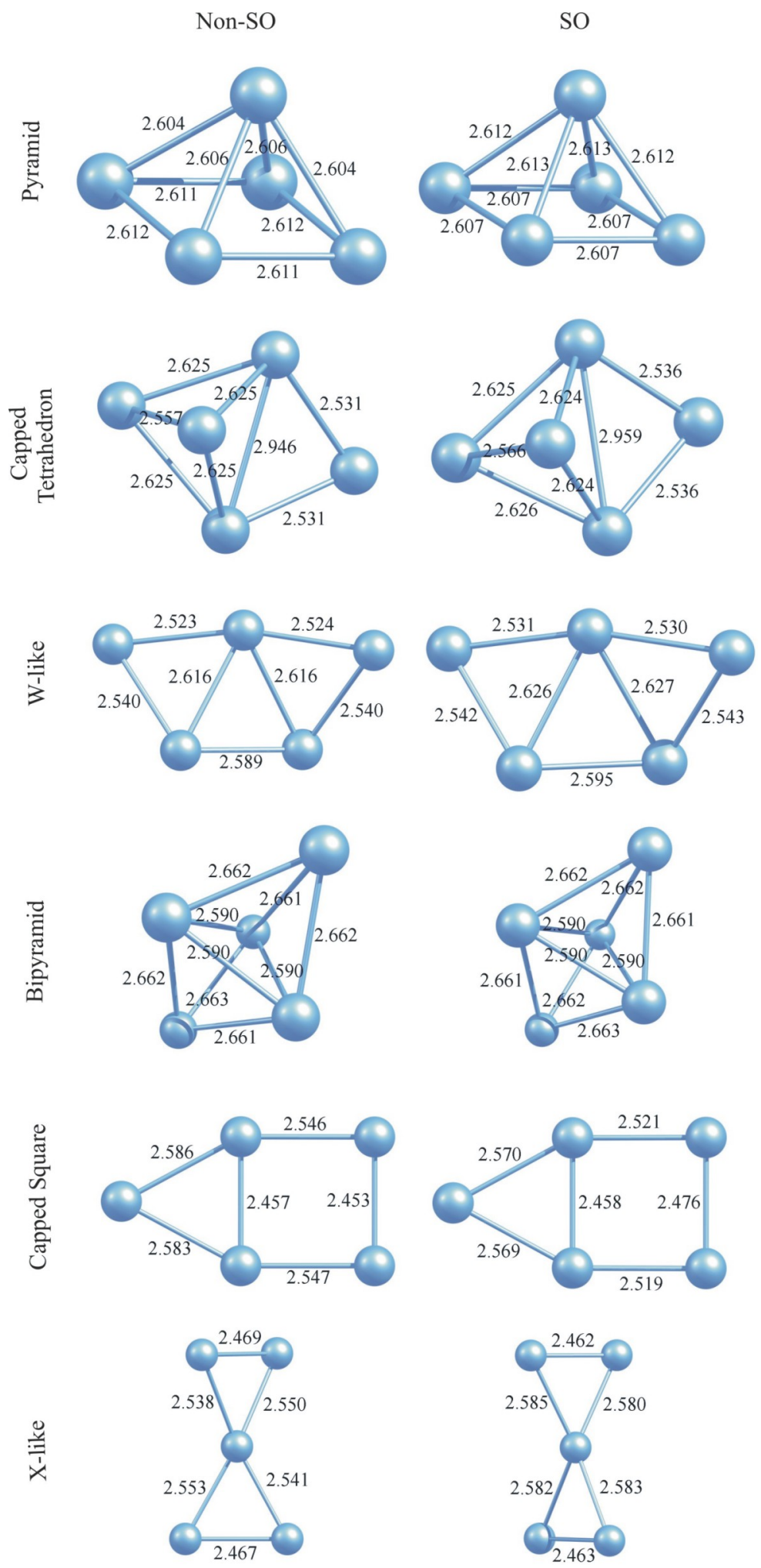

FIG. 2: Relaxed geometries of $\mathrm{Pt}_{5}$ isomers for the most stable spin multiplicity of each isomer with and without SO coupling effects (distances are in $\stackrel{\circ}{A}$ ). 

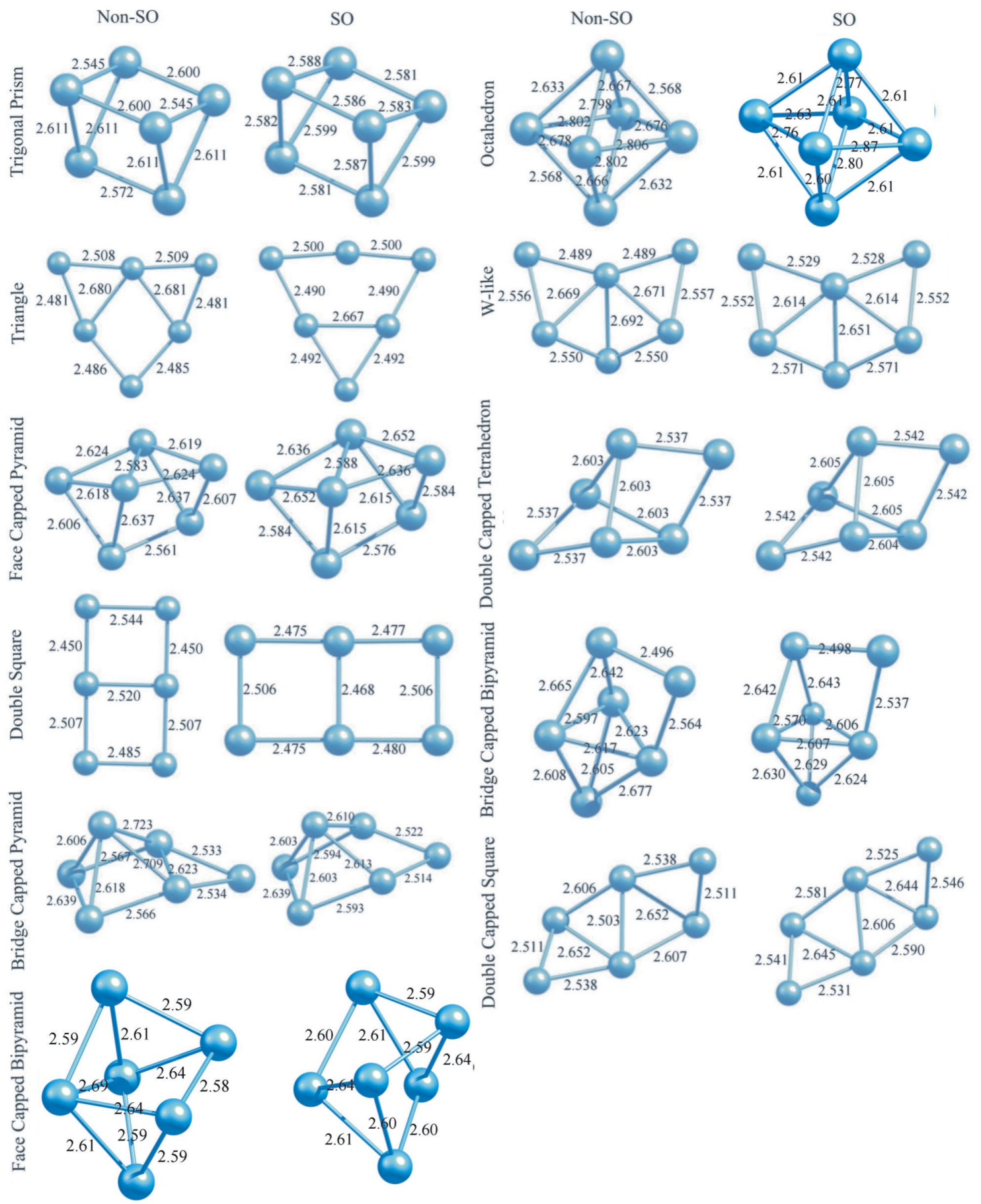

FIG. 3: Relaxed geometries of $\mathrm{Pt}_{6}$ isomers for the most stable spin multiplicity of each isomer with and without SO coupling effects (distances are in $\stackrel{A}{A}$ ). 